\title{
Interlaboratory Comparison of Magnetic Thin Film Measurements
}

\begin{tabular}{ll}
\hline Volume 108 & \multicolumn{1}{c}{ Number 2} \\
\hline $\begin{array}{l}\text { F. C. S. da Silva, C. M. Wang, } \\
\text { and D. P. Pappas }\end{array}$ & $\begin{array}{l}\text { A potential low magnetic moment standard } \\
\text { reference material (SRM) was studied in } \\
\text { an interlaboratory comparison. The mean } \\
\text { and the standard deviation of the satura- } \\
\text { tion moment } m_{\mathrm{s}} \text {, the remanent moment } m_{\mathrm{r}}\end{array}$ \\
and the intrinsic coercivity $H_{\mathrm{c}}$ of nine sam- \\
ational Institute of Standards \\
and Technology, \\
$\begin{array}{l}\text { ples were extracted from hysteresis-loop } \\
\text { measurements. Samples were measured by } \\
\text { thirteen laboratories using inductive-field } \\
\text { Boulder, CO 80305 } \\
\text { loopers, vibrating-sample magnetometers, } \\
\text { alternating-gradient force magnetometers, } \\
\text { and superconducting quantum-interfer- } \\
\text { ence-device magnetometers. NiFe films on } \\
\text { Si substrates had saturation moment meas- } \\
\text { jwang@boulder.nist.gov } \\
\text { pappas@boulder..nist.gov }\end{array}$ \\
$\begin{array}{l}\text { amonts reproduced within } 5 \% \text { variation } \\
\text { among the laboratories. The results show }\end{array}$
\end{tabular}

March-April 2003

\author{
that a good candidate for an SRM must \\ have a highly square hysteresis loop \\ $\left(m_{\mathrm{r}} / m_{\mathrm{s}}>90 \%\right), H_{\mathrm{c}} \approx 400 \mathrm{~A} \cdot \mathrm{m}^{-1}(5 \mathrm{Oe})$, \\ and $m_{\mathrm{s}} \approx 2 \times 10^{-7} \mathrm{~A} \cdot \mathrm{m}^{2}\left(2 \times 10^{-4} \mathrm{emu}\right)$.
}

Key words: interlaboratory comparison; magnetic films; standard reference materials.

Accepted: January 24, 2003

Available online: http://www.nist.gov/jres

\section{Introduction}

Control of a wide range of magnetic properties is critical in the manufacturing of magnetic data-storage devices. These properties include, among others, the saturation and remanent magnetic moments, and the intrinsic coercivity. The desired values for these properties are specific to the particular application and can cover a wide range. This is illustrated in a data storage system, based on magnetic tape or hard disk drives, where thin films with low intrinsic coercivities are used in the read heads while relatively thick films with high moments and high intrinsic coercivities are used for the storage media. These devices can involve multiple layers of various magnetic and nonmagnetic materials composed of an assortment of alloys.

In addition, an important problem in the technology and manufacturing of magnetic thin film devices is the determination of the film thickness. This goes beyond the element-specific (e.g., $\mathrm{Fe}, \mathrm{Ni}$, and $\mathrm{Co}$ ) thickness calibration because the relevant properties of devices using this technology are dominated by the interfaces. These properties are affected by intermixing and alloying, pinholes, reduced atomic coordination, and quantum-well effects. These effects are in turn determined by growth temperature, composition modulation, substrate strain, and microscopic morphology of the films. Therefore, it is necessary to measure the actual magnetic properties of samples as deposited in order to correlate useful properties (magnetoresistance, intrinsic coercivity, anisotropy) with magnetic moments. Recent developments in technology that use magnetic layers less than $10 \mathrm{~nm}$ thick, e.g., giant magnetoresistance (GMR) sensors with correspondingly low moments (on the order of $10^{-8} \mathrm{~A} \cdot \mathrm{m}^{2}$ ), present stringent requirements on process and quality control.

Therefore the calibration of magnetic-property measurement techniques at the lowest range is important for process and quality control as well as for research. Because most of the measurement techniques currently in use are sensitive to the fields generated by the sample, they are also sensitive to the sample geom- 
etry. Hence, care must be taken to choose a calibration reference artifact that has both a small moment and the same form factor (thin film geometry) as the samples to be measured. At present, NIST offers two standard reference materials (SRMs) for magnetometer calibration: SRM 762 and SRM 772a. Both SRMs have magnetic moments in the scale of $10^{-3} \mathrm{~A} \cdot \mathrm{m}^{2}(1 \mathrm{emu})$.

The objective of this study was to investigate the calibration needs of the magnetic recording industry and identify the greatest need for standard reference samples. This required evaluating several measurement techniques, magnetic properties, and samples. We concentrated on a few common measurement techniques and low moment samples, similar to those used in magnetoresistive read heads.

\section{Methods}

An interlaboratory comparison study for magnetic characterization was undertaken in which 9 ferromagnetic samples were sent to 13 laboratories from the magnetic recording industry, NIST, academia, and magnetic instrument manufacturers. The samples were circulated in a serial fashion, with each laboratory allotted approximately 3 days for measurements. Table 1 shows the composition and dimensions of the 9 samples.

Table 1. Samples used in the study

\begin{tabular}{ccc}
\hline \hline Sample & Composition & $\begin{array}{c}\text { Nominal magnetic } \\
\text { film dimensions }\end{array}$ \\
\hline $\mathrm{A}$ & $\mathrm{Si} / \mathrm{Ta} / \mathrm{NiFe} / \mathrm{Ta}$ & $\pi \times(38 \mathrm{~mm})^{2} \times 10 \mathrm{~nm}$ \\
$\mathrm{~B}$ & $\mathrm{Si} / \mathrm{Ta} / \mathrm{NiFe} / \mathrm{Ta}$ & $\pi \times(38 \mathrm{~mm})^{2} \times 5 \mathrm{~nm}$ \\
$\mathrm{C}$ & $\mathrm{Si} / \mathrm{Ta} / \mathrm{NiFe} / \mathrm{Ta}$ & $\pi \times(38 \mathrm{~mm})^{2} \times 2.5 \mathrm{~nm}$ \\
$\mathrm{D}$ & $\mathrm{Si} / \mathrm{Ta} / \mathrm{NiFe} / \mathrm{Ta}$ & $5 \mathrm{~mm} \times 5 \mathrm{~mm} \times 10 \mathrm{~mm}$ \\
$\mathrm{E}$ & $\mathrm{Si} / \mathrm{Ta} / \mathrm{NiFe} / \mathrm{Ta}$ & $5 \mathrm{~mm} \times 5 \mathrm{~mm} \times 5 \mathrm{~mm}$ \\
F & $\mathrm{Si} / \mathrm{Ta} / \mathrm{NiFe} / \mathrm{Ta}$ & $5 \mathrm{~mm} \times 5 \mathrm{~mm} \times 2.5 \mathrm{~mm}$ \\
$\mathrm{G}$ & $\mathrm{C}(100) / \mathrm{Ni}(100) / \mathrm{Cu}(100)$ & $3 \mathrm{~mm} \times 2 \mathrm{~mm} \times 25 \mathrm{~mm}$ \\
$\mathrm{H}$ & $\mathrm{C}(100) / \mathrm{Ni}(100) / \mathrm{Cu}(100)$ & $3 \mathrm{~mm} \times 2 \mathrm{~mm} \times 10 \mathrm{~mm}$ \\
$\mathrm{I}$ & $\mathrm{C}(100) / \mathrm{Ni}(100) / \mathrm{Cu}(100)$ & $3 \mathrm{~mm} \times 2 \mathrm{~mm} \times 2.5 \mathrm{~mm}$ \\
\hline
\end{tabular}

Two different types of samples were prepared. The first type was composed of a Permalloy $\left(\mathrm{Ni}_{81} \mathrm{Fe}_{19}\right)$ film sandwiched between Ta layers. These samples are similar to the free magnetic layer used in magnetoresistive (MR) heads in terms of thickness, magnetization, total moment, intrinsic coercivity, and geometry. Wafers 7.5 $\mathrm{cm}$ in diameter were used for samples $\mathrm{A}, \mathrm{B}$, and $\mathrm{C}$ because the first step in the head manufacturing process requires quality control at the wafer level. Smaller sizes were used for samples D, E, and F because the second step in quality control is typically to dice wafers and study them at the coupon level with high-field magnetometers. These two sample geometries allowed us to make direct connections to relevant processes in head metrology. The second type of samples were ultra-thin single-crystal Ni films grown on diamond substrates and capped with $\mathrm{Cu}$. These samples have well characterized magnetic and structural properties [1-7] and moments comparable to those of samples used for head metrology.

Four types of magnetic measurement tools were used in this study: vibrating-sample magnetometer (VSM) $[8,9]$, alternating gradient force magnetometer (AGM) [10-12], superconducting quantum-interference-device (SQUID) magnetometer [13], and inductive-field (B-H) looper [14]. In practice, these tools fall into two general categories: magnetometric and fluxmetric. In magnetometric systems (AGM, VSM, and SQUID), the magnetic field generated by the sample (approximated by a dipole) is measured. In fluxmetric systems (B-H loopers) the fields inside the sample are measured by directly measuring the flux variations around a cross section of the sample.

The tools in the first category (VSM, AGM, and SQUID) are generally used with small samples (dimensions on the order of $1 \mathrm{~cm}$ ) that are suspended on a long, slender rod. The rod is adjusted so the sample is positioned near a relatively large pickup loop or modulating coil. This category of magnetometers has the advantage that the sample can be placed between the poles of an electromagnet. However, the physical position, sample size, and mounting procedures are very important. If these problems are addressed correctly, this first category can be calibrated in terms of the total moment of the sample.

In the second category of tools (B-H loopers), the sample is held in a rigid fixture inside a coreless magnetizing coil with a relatively short, close-fitting inductive sense coil. This reduces the sensitivity of the measurement to sample alignment. In general, B-H loopers are useful for thin-film samples of large area and are used to screen entire wafers in quality control. In this geometry, the measured quantity is the flux enclosed by the sense coil.

Without making assumptions about sample shape, homogeneity, or field distribution, it is not possible to directly analyze the results of the two categories of measurement tools. In this study, therefore, we focused on the consistency between relative measurements of two different samples. For calibrated measurements, measured voltages have to be scaled by the voltage corresponding to a standard reference material. This means 
that the absolute quantities reported in this paper reflect the reproducibility of the laboratories' own measurements.

A form was sent with the samples to ensure uniform reporting of results. Information to be entered included the laboratory, operator, date, sample measured, and technique used. The section for the results requested measurements of the total saturation moment $m_{\mathrm{s}}$, total remanent moment $m_{\mathrm{r}}$, and intrinsic coercivity $H_{\mathrm{c}}$. Finally, the back side of the form contained instructions for handling the sample and a table for the operator to record the measurement parameters (e.g., maximum field, sweep rates, integration time constants.)

\section{Results}

Figures 1, 2, 3, and 4 show examples of hysteresis curves obtained by the participating laboratories using the four different measurement techniques. For all curves, the signal-to-noise ratio $(S N R)$ was obtained by taking the amplitude of the total curve $\left(\approx 2 m_{\mathrm{s}}\right)$ and dividing by the standard uncertainty of the noise after saturation $\left[\sqrt{\left\langle\delta^{2} m\right\rangle}\right]$. The estimates of uncertainties for $m_{\mathrm{s}}$ and $m_{\mathrm{r}}$ were defined as the reciprocal of the $S N R$.

Figure 1 shows a hysteresis curve measured on sample A using a B-H looper. Here, the measurement was performed at a field frequency of $2 \mathrm{~Hz}$ with 10 averages used to obtain the final curve. Notice that the magnetic flux $\Phi$ is reported instead of the magnetic moment $m$. This did not affect the comparison between samples A, $\mathrm{B}$, and $\mathrm{C}$ since they were measured only with $\mathrm{B}-\mathrm{H}$ loopers. Since $S N R_{\mathrm{BH}}=1000$ for Fig. 1, we report the fixed uncertainty in $m_{\mathrm{s}}$ and $m_{\mathrm{r}}$ for B-H loopers as $0.1 \%$.

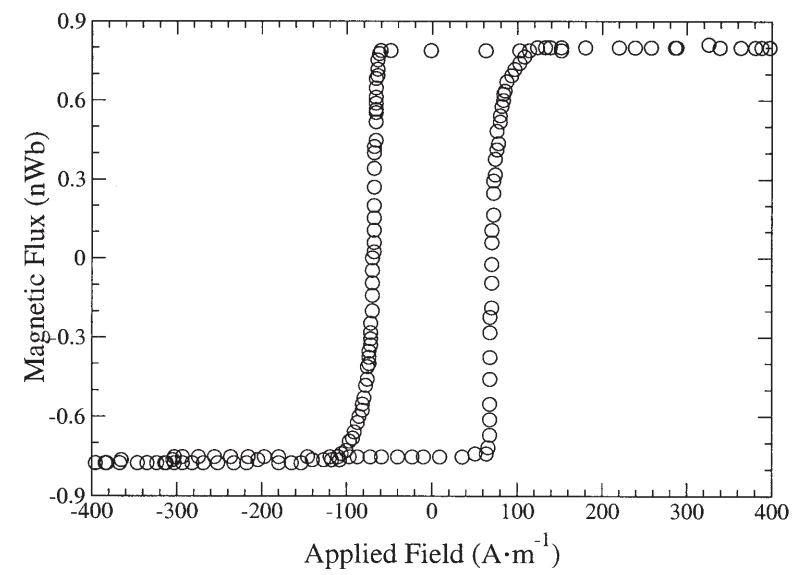

Fig. 1. Hysteresis curve of sample A measured with a B-H looper.
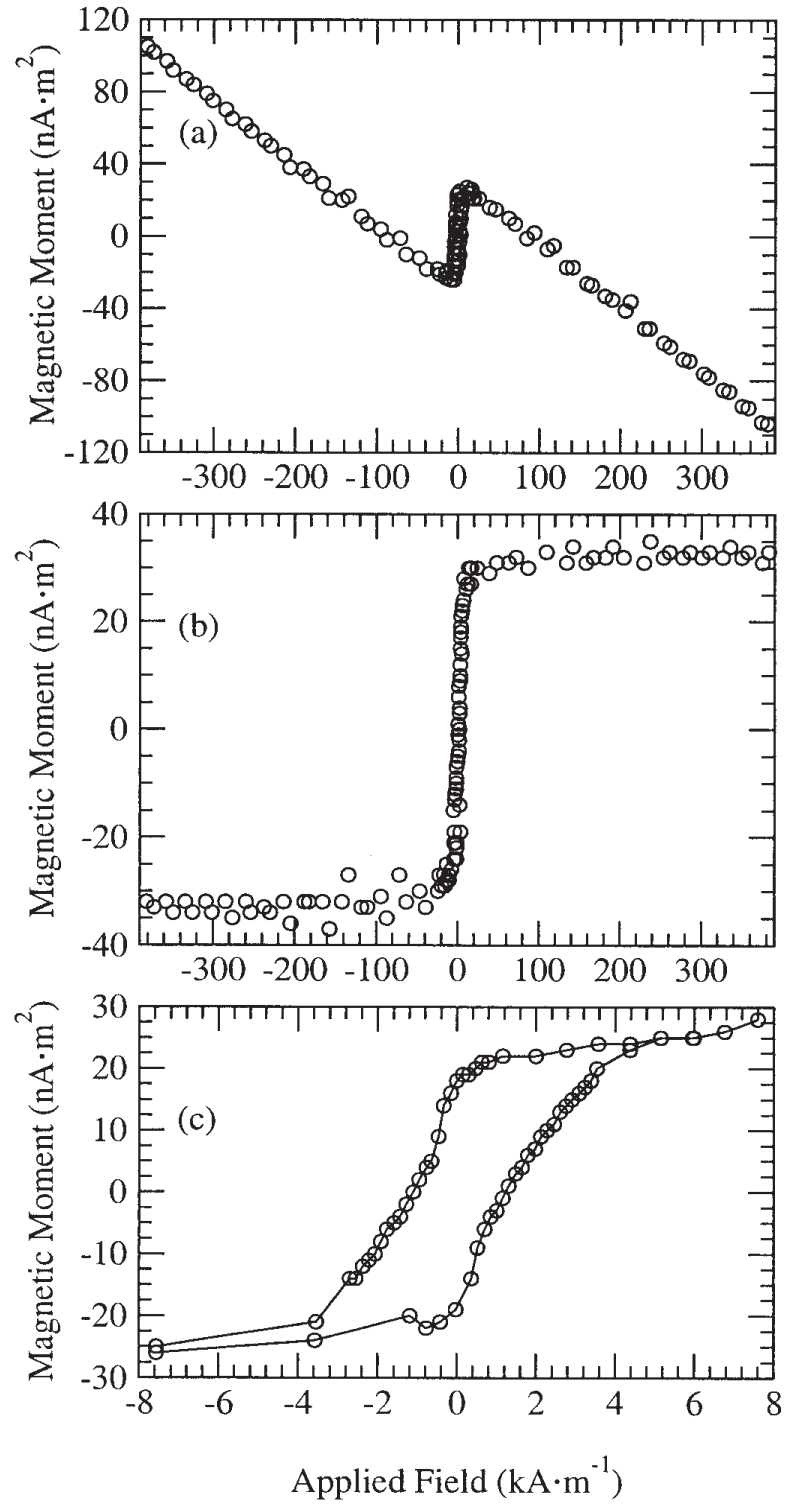

Fig. 2. Hysteresis curve of sample $H$ measured with a SQUID, showing the data (a) before and (b-c) after the diamagnetic subtraction.

Figure 2(a) shows the hysteresis curve of sample $\mathrm{H}$ measured with a SQUID magnetometer. Here, the time interval between points on the curve was $200 \mathrm{~s}$. The raw data show the diamagnetic contribution due to the diamond substrate. This dramatic effect is a combination of the diamagnetic susceptibility of diamond, which is $50 \%$ greater than that of $\mathrm{Si}$, and the low mass ratio between the Ni film and the diamond substrate. The result after the subtraction of the diamagnetic contribution is shown in Fig. 2(b), from which we obtain $S N R_{\text {SQUID }}=16$. This $S N R$ gives an uncertainty in the 


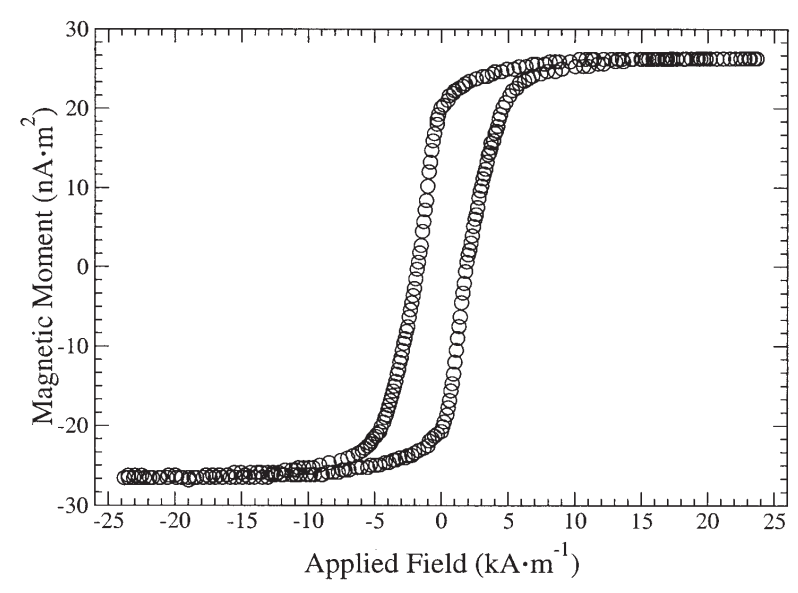

Fig. 3. Hysteresis curve of sample $\mathrm{H}$ measured with an AGM.

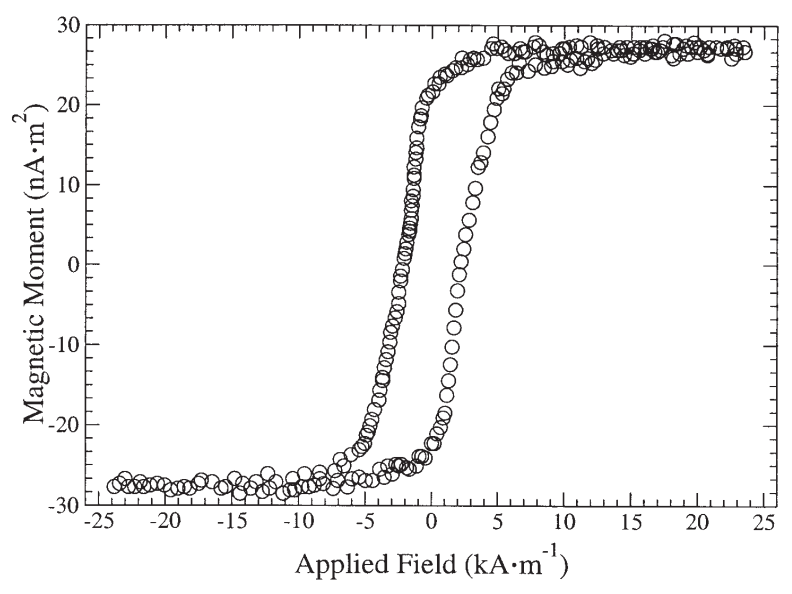

Fig. 4. Hysteresis curve of sample H measured with an VSM.

magnetic moment of $6.3 \%$. Figure 2 (c) shows the same corrected curve where better resolution around zero applied field leads to estimates of the remanence and intrinsic coercivity.

Figure 3 shows a hysteresis curve of sample $\mathrm{H}$ measured with an AGM. The time between points in this measurement was $1 \mathrm{~s}$. The curve does not show the diamagnetic contribution because lower magnetic fields were applied. Here, $S N R_{\mathrm{AGM}}=54$, giving an uncertainty in the magnetic moment of $1.9 \%$.

Figure 4 shows the hysteresis curve of sample $\mathrm{H}$ measured with a VSM. At an interval of $1 \mathrm{~s}$ between measurements, we have $S N R_{\mathrm{VSM}}=15$, yielding an uncertainty of $6.7 \%$ in the magnetic moment.

The hysteresis curves were used to extract three quantities: the saturation moment $m_{\mathrm{s}}$, the remanent moment $m_{\mathrm{r}}$, and the intrinsic coercivity $H_{\mathrm{c}}$. Figures 5, 6, and 7, respectively present the measured values of $m_{\mathrm{s}}$, $m_{\mathrm{r}}$, and $H_{\mathrm{c}}$ for all nine samples and all four measurement techniques. Some samples were measured more than once by the same laboratory using the same technique. In these cases, the reported quantity is the average of two measurements of the same quantity. All SQUID measurements, on the other hand, provided only one measurement of $m_{\mathrm{s}}$ and $m_{\mathrm{r}}$.

A simple rule for outlying points was based on the report of each laboratory on how the measurements were done. A few measurements were discarded when the calibration procedure did not follow the procedure used by the other laboratories. However, all relative measurements (e.g., $m_{\mathrm{s}} / m_{\mathrm{r}}$ ratio) could be used since the effect of calibration factors was minimized.

Figure 8 shows the ratio $m_{\mathrm{s}} / m_{\mathrm{r}}$ obtained using the data shown in Figs. 5 and 6. Absolute B-H looper measurements on samples D and E could not be compared with the other techniques for lack of an unambiguous procedure to convert from magnetic flux to magnetic moment. However, it was still justifiable to compare the ratios $m_{\mathrm{s}} / m_{\mathrm{r}}$ and $m_{\mathrm{s}}^{\text {sampleE }} / m_{\mathrm{s}}^{\text {sampleD }}$ (Figs. 8 and 9), since the relative conversion factors cancel.

Each absolute quantity measured for a given sample using a particular technique by different laboratories formed a set. The mean and the standard uncertainty of each set were calculated. The reproducibility parameter here was calculated as the standard uncertainty of the quantities in each set. Tables 2-4 list the means and the standard uncertainties of the saturation and remanent moments and the intrinsic coercivity across the laboratories. The ratio of the remanent-to-saturation moment of each sample and the saturation moment ratio between samples is shown in Tables 5 and 6 .

\section{Discussion}

The main goal of this interlaboratory comparison was to identify standard procedures and materials that could provide reproducible laboratory measurements. Due to a few non-conformities, a precise analysis of the statistical data [15] was not possible. The specified measurement procedures were not followed exactly by all the participants. Also, not all quantities requested in the form were measured twice to provide uncertainties to the measurements. In the laboratories' reports, samples $\mathrm{F}$ and I showed initial stages of oxidation, which compromises any conclusion about these samples. However, the data showed enough statistical validity to determine the main features for a low magnetic moment SRM candidate. 

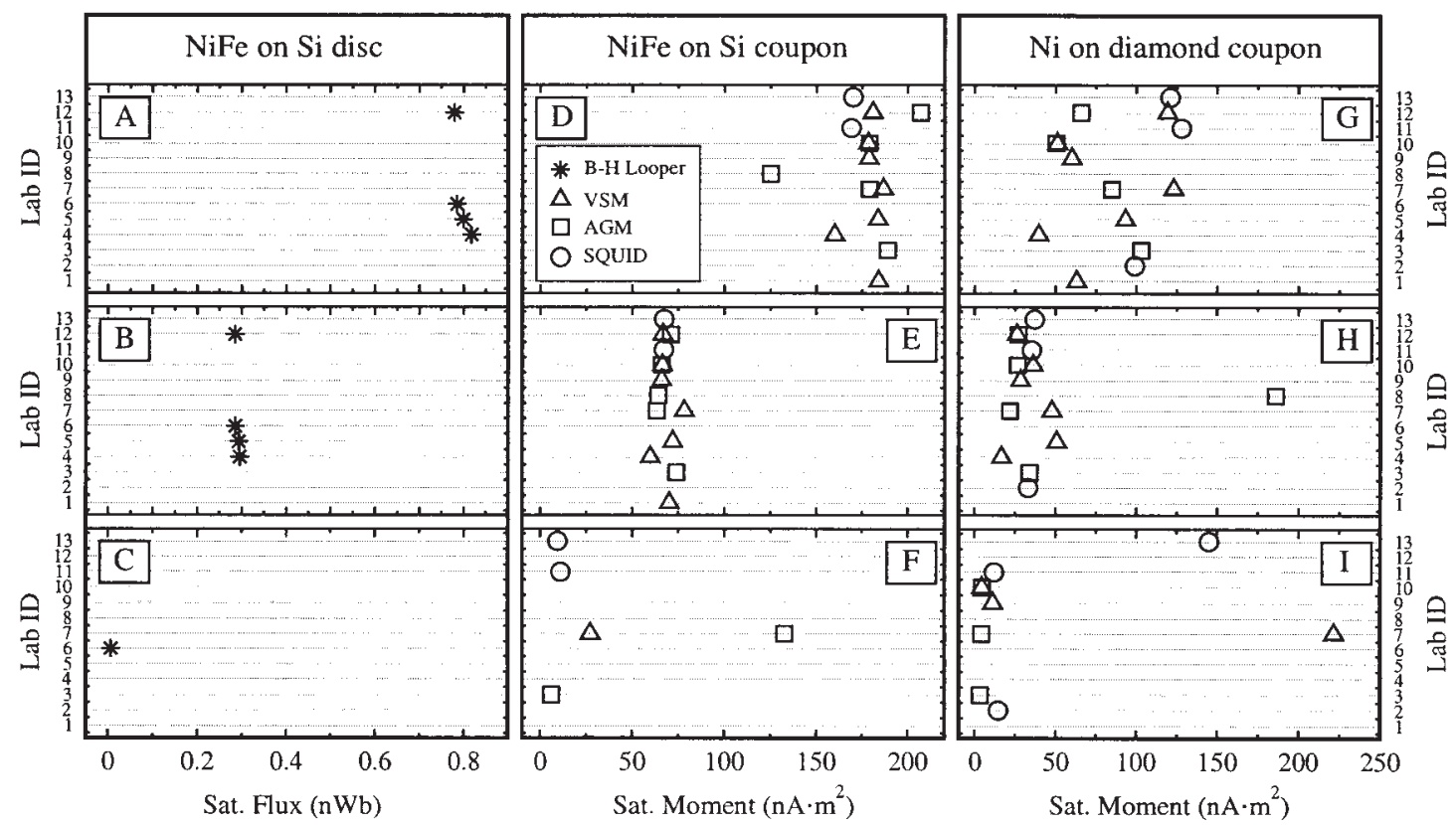

Fig. 5. Interlaboratory comparison data showing the absolute values of the saturation moment $m_{\mathrm{s}}$. B-H looper measurements of flux on samples D and E are shown in Table 2.
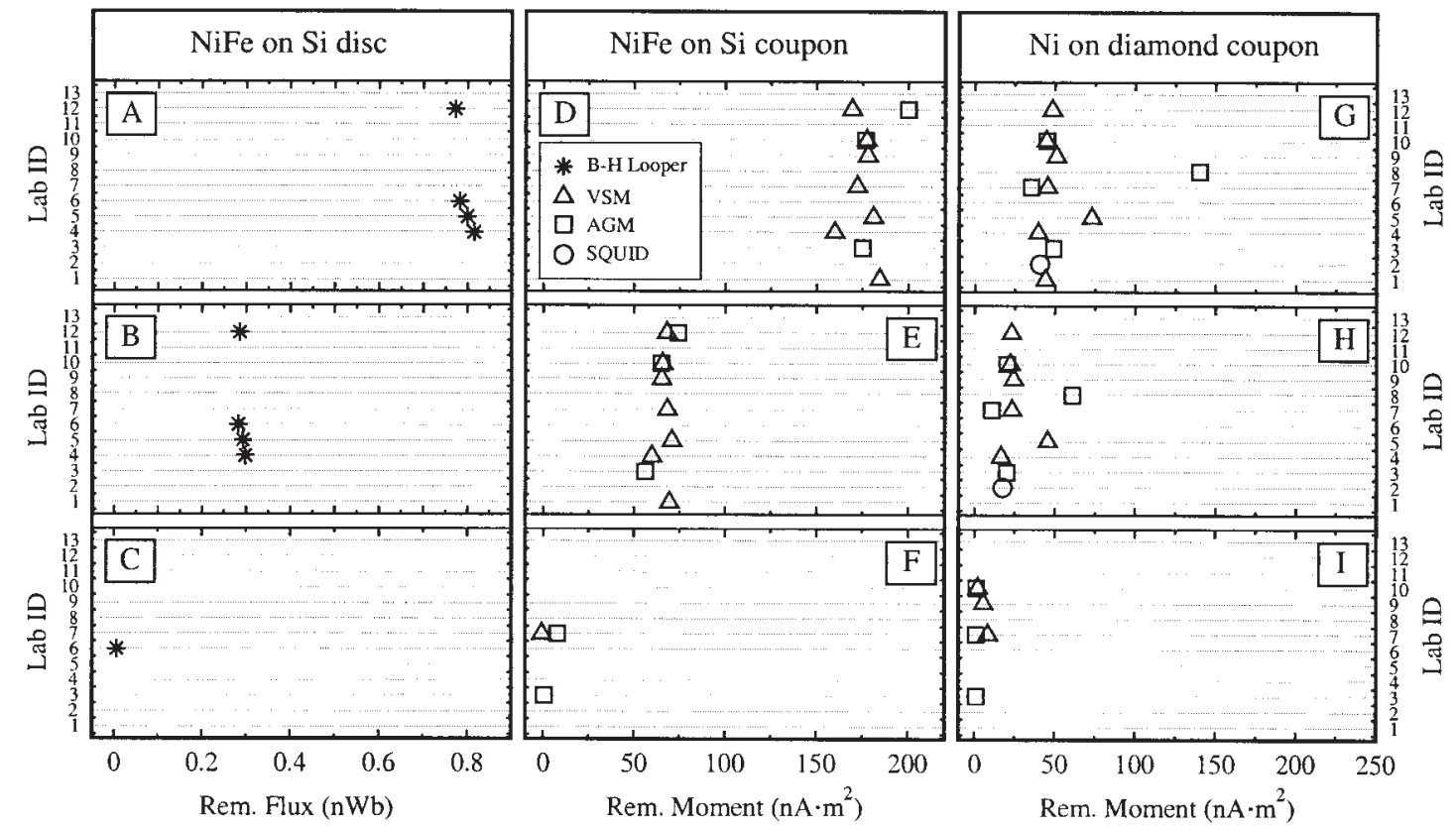

Fig. 6. Interlaboratory comparison data showing the values of the remanent moment. B-H looper measurements of flux on samples $\mathrm{D}$ and $\mathrm{E}$ are shown in Table 3. 


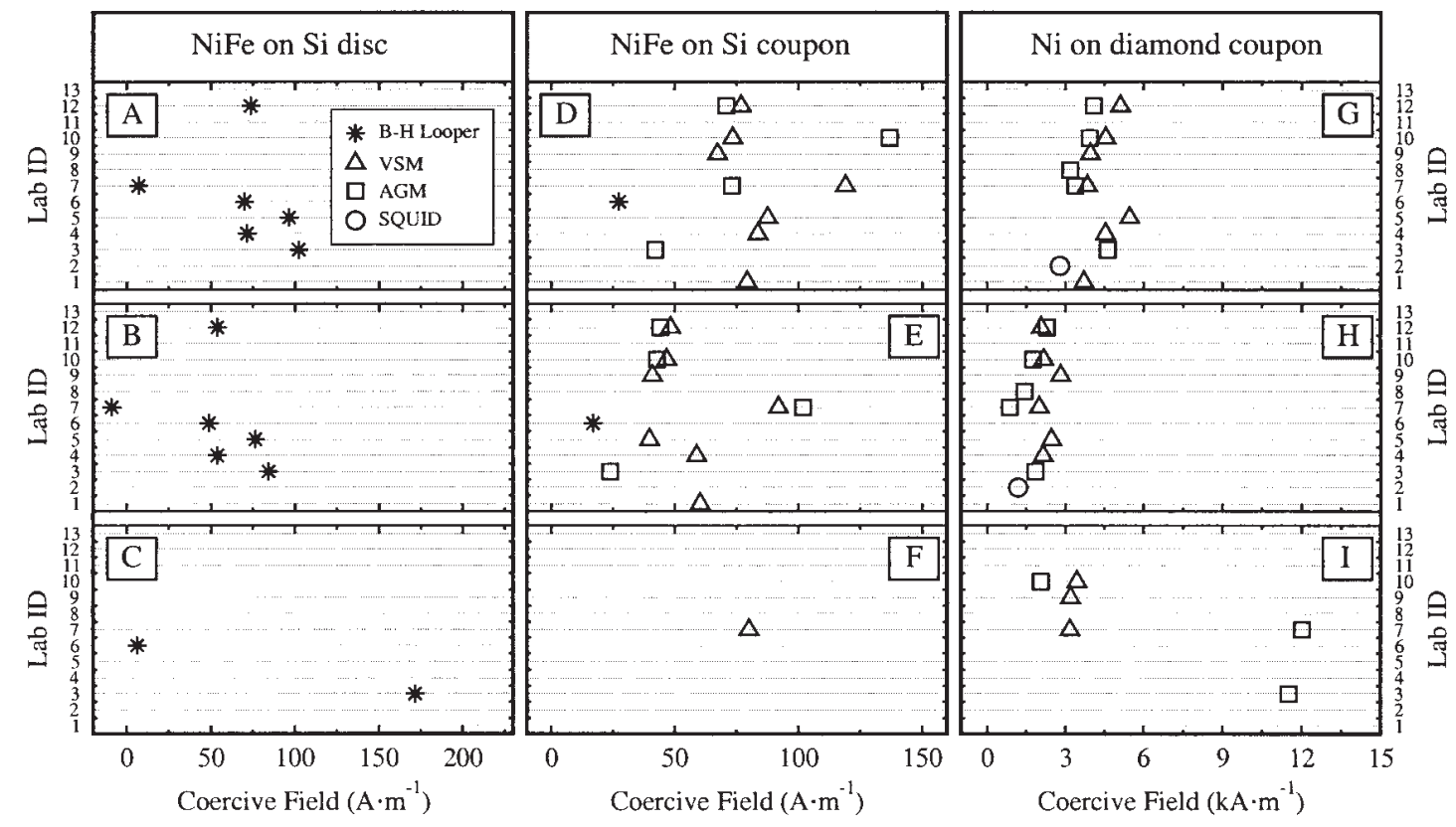

Fig. 7. Interlaboratory comparison data showing the values of the intrinsic coercivity.

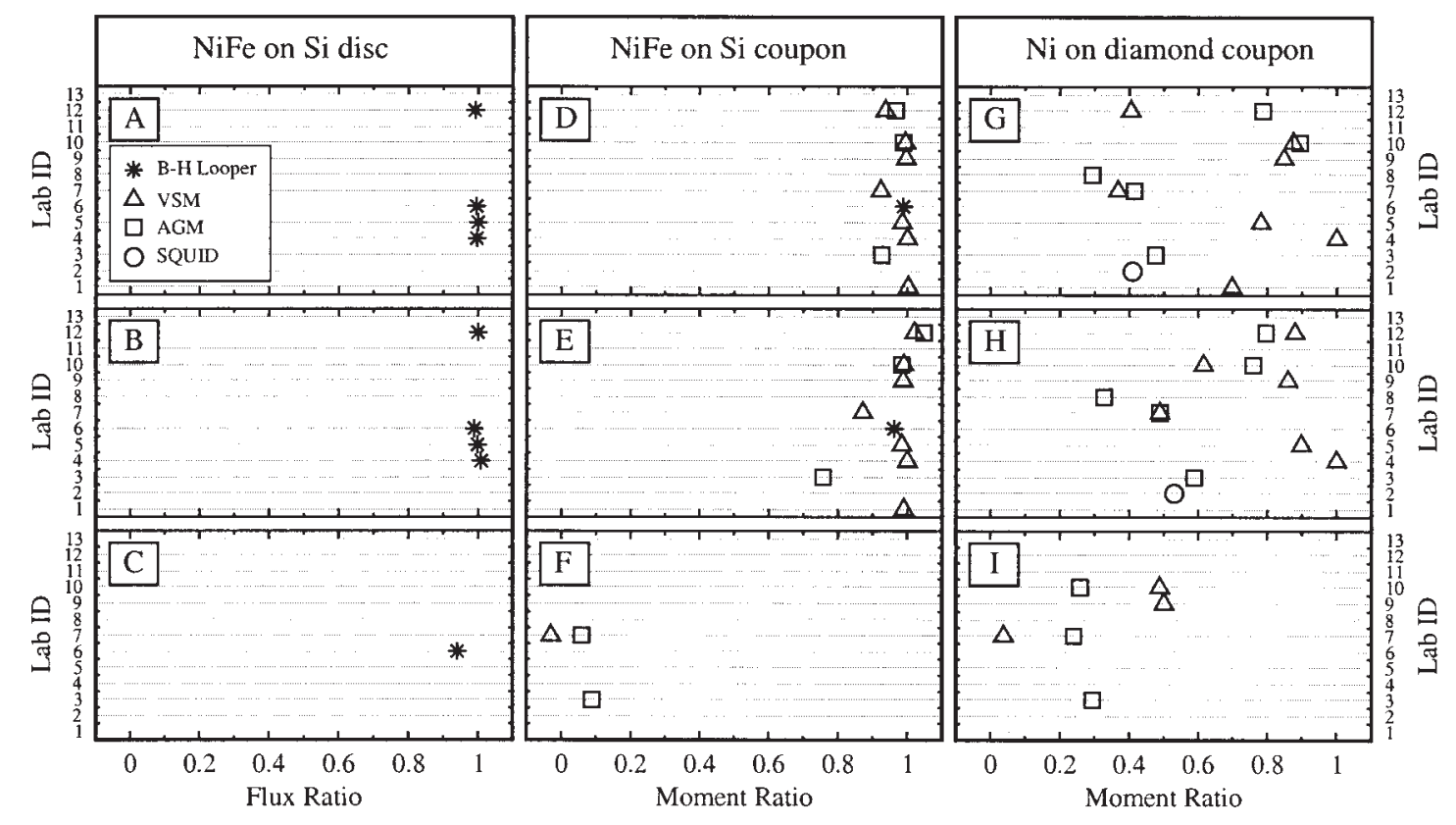

Fig. 8. Interlaboratory comparison data showing the remanence-to-saturation ratio for each sample. 


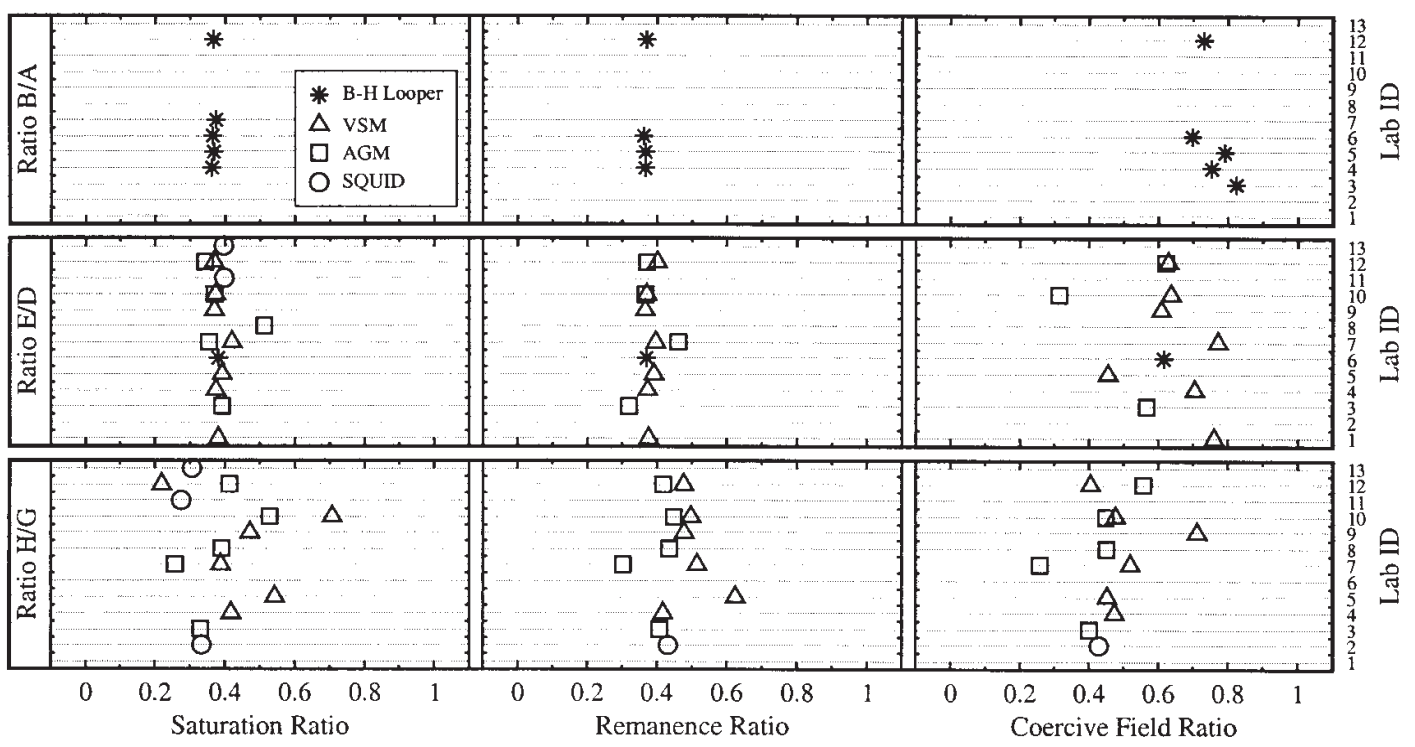

Fig. 9. Interlaboratory comparison data showing the intralaboratory ratios of the saturation moments, the remanent moments, and the intrinsic coercivities between two samples.

Table 2. Statistical analysis of the saturation moment. In each cell, the top values are the mean and the bottom values are the standard uncertainties divided by the corresponding means

\begin{tabular}{|c|c|c|c|c|c|c|c|c|c|}
\hline \multicolumn{10}{|c|}{ Saturation flux (pWb) } \\
\hline Method & $\mathrm{A}$ & $\mathrm{B}$ & $\mathrm{C}$ & $\mathrm{D}$ & $\mathrm{E}$ & $\mathrm{F}$ & G & $\mathrm{H}$ & I \\
\hline \multirow[t]{2}{*}{$\mathrm{BH}$} & 795.3 & 290.1 & 6.8 & 55.5 & 21.1 & & & & \\
\hline & $2.2 \%$ & $1.8 \%$ & & & & & & & \\
\hline \multicolumn{10}{|c|}{ Saturation moment $\left(\mathrm{nA} \cdot \mathrm{m}^{2}\right)$} \\
\hline Method & $\mathrm{A}$ & $\mathrm{B}$ & $\mathrm{C}$ & $\mathrm{D}$ & $\mathrm{E}$ & $\mathrm{F}$ & G & $\mathrm{H}$ & I \\
\hline \multirow[t]{2}{*}{ VSM } & & & & 179 & 68 & 27 & 78 & 34 & 79 \\
\hline & & & & $5 \%$ & $9 \%$ & & $44 \%$ & $38 \%$ & $160 \%$ \\
\hline \multirow[t]{2}{*}{ AGM } & & & & 176 & 68 & 69 & 76.0 & 59 & 4 \\
\hline & & & & $17 \%$ & $7 \%$ & $130 \%$ & $30 \%$ & $120 \%$ & $15 \%$ \\
\hline \multirow[t]{2}{*}{ SQUID } & & & & 169.5 & 67.2 & 10.2 & 83.0 & 24.1 & 52.3 \\
\hline & & & & $0.4 \%$ & $0.1 \%$ & $12 \%$ & $87 \%$ & $87 \%$ & $150 \%$ \\
\hline
\end{tabular}

Magnetic flux for samples A, B, and C (Permalloy on Si wafer) could be measured with an uncertainty of $3 \%$. This result shows that SRMs in the shape of wafers are useful for measurements of saturation and remanence. A procedure similar to that used for calibrating magnetometric measurement systems needs to be developed in order for comparisons (after a wafer dicing, for example) to be done in moment units.
The diamagnetism in some reported magnetometric hysteresis curves is an undesired source of uncertainty. Since low magnetic moment samples are usually thin films on bulk substrates, SRMs have to be specified with low intrinsic coercivity to minimize diamagnetic effects due to the substrate. Also, to ensure that the magnetic film is a monodomain in the saturated state, the SRMs have to be specified with high squareness 
Table 3. Statistical analysis of the remanent moment. In each cell, the top values are the mean and the bottom values are the standard uncertainties divided by the corresponding means

\begin{tabular}{|c|c|c|c|c|c|c|c|c|c|}
\hline \multicolumn{10}{|c|}{ Remanent flux (pWb) } \\
\hline Method & $\mathrm{A}$ & $\mathrm{B}$ & $\mathrm{C}$ & $\mathrm{D}$ & $\mathrm{E}$ & $\mathrm{F}$ & G & $\mathrm{H}$ & I \\
\hline \multirow[t]{2}{*}{$\mathrm{BH}$} & 792.0 & 289.9 & 6.4 & 54.9 & 20.3 & & & & \\
\hline & $2.4 \%$ & $2.4 \%$ & & & & & & & \\
\hline \multicolumn{10}{|c|}{ Remanent moment $\left(\mathrm{nA} \cdot \mathrm{m}^{2}\right)$} \\
\hline Method & A & $\mathrm{B}$ & $\mathrm{C}$ & $\mathrm{D}$ & $\mathrm{E}$ & $\mathrm{F}$ & G & $\mathrm{H}$ & I \\
\hline \multirow[t]{2}{*}{ VSM } & & & & 175 & 67 & & 49 & 26 & 5 \\
\hline & & & & $46 \%$ & $6 \%$ & & $22 \%$ & $38 \%$ & $60 \%$ \\
\hline \multirow[t]{2}{*}{ AGM } & & & & 183.9 & 65.0 & 4.2 & 45.2 & 18.1 & 1.0 \\
\hline & & & & $76 \%$ & $14 \%$ & $120 \%$ & $16 \%$ & $28 \%$ & $1 \%$ \\
\hline SQUID & & & & & & & 40.5 & 17.5 & \\
\hline
\end{tabular}

Table 4. Statistical analysis of the intrinsic coercivity. In each cell, the top values are the mean and the bottom values are the standard uncertainties divided by the corresponding means

\begin{tabular}{|c|c|c|c|c|c|c|c|c|c|}
\hline \multicolumn{10}{|c|}{ Intrinsic coercivity $\left(\mathrm{A} \cdot \mathrm{m}^{-1}\right)$} \\
\hline Method & A & $\mathrm{B}$ & $\mathrm{C}$ & $\mathrm{D}$ & $\mathrm{E}$ & $\mathrm{F}$ & G & $\mathrm{H}$ & I \\
\hline \multirow[t]{2}{*}{$\mathrm{BH}$} & 70 & 51 & 86 & 28 & 17 & & & & \\
\hline & $49 \%$ & $65 \%$ & $27 \%$ & & & & & & \\
\hline \multirow[t]{2}{*}{ VSM } & & & & 84 & 55 & 80 & 4451 & 2277 & 3272 \\
\hline & & & & $20 \%$ & $35 \%$ & & $15 \%$ & $14 \%$ & $5 \%$ \\
\hline \multirow[t]{2}{*}{ AGM } & & & & 81 & 53 & 1576 & 3836 & 1637 & 8527 \\
\hline & & & & $50 \%$ & $60 \%$ & $110 \%$ & $15 \%$ & $32 \%$ & $66 \%$ \\
\hline SQUID & & & & & & & 2785 & 1194 & \\
\hline
\end{tabular}

Table 5. Statistical analysis of the remanent-to-saturation moment ratio. In each cell, the top values are the mean and the bottom values are the standard uncertainties divided by the corresponding means

\begin{tabular}{|c|c|c|c|c|c|c|c|c|c|}
\hline \multicolumn{10}{|c|}{ Remanent to saturation moment ratio } \\
\hline Method & A & B & $\mathrm{C}$ & $\mathrm{D}$ & E & F & G & $\mathrm{H}$ & I \\
\hline \multirow[t]{2}{*}{$\mathrm{BH}$} & 0.996 & 0.999 & 0.941 & 0.988 & 0.962 & & & & \\
\hline & $0.3 \%$ & $0.8 \%$ & & & & & & & \\
\hline \multirow[t]{2}{*}{ VSM } & & & & 0.98 & 0.98 & & 0.71 & 0.79 & 0.26 \\
\hline & & & & $0.3 \%$ & $0.5 \%$ & & $34 \%$ & $25 \%$ & $100 \%$ \\
\hline \multirow[t]{2}{*}{$\mathrm{AGM}$} & & & & 0.73 & 0.70 & 0.07 & 0.57 & 0.59 & 0.26 \\
\hline & & & & $64 \%$ & $67 \%$ & $29 \%$ & $46 \%$ & $32 \%$ & $12 \%$ \\
\hline SQUID & & & & & & & 0.41 & 0.53 & \\
\hline
\end{tabular}


Table 6. Statistical analysis of the ratio of magnetic quantities of two samples. In each cell, the top values are the mean and the bottom values are the standard uncertainties divided by the corresponding means

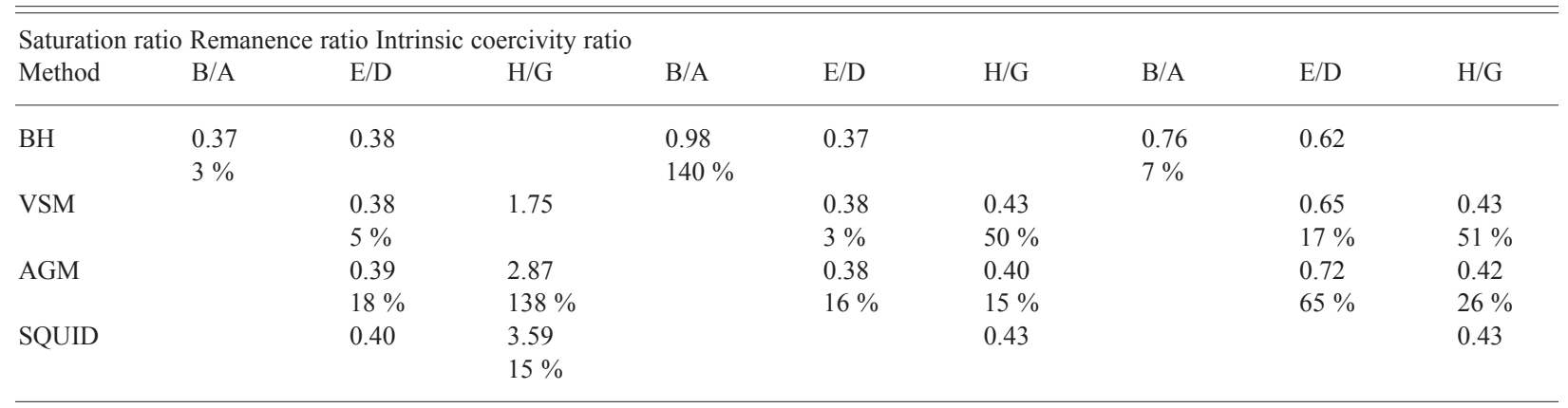

$\left(m_{\mathrm{r}} / m_{\mathrm{s}} \approx 1\right)$. These specifications were confirmed by saturation measurements performed on samples D and E (Permalloy on Si coupon), which showed mean standard uncertainties of $8 \%$ and $5 \%$, respectively.

The measurements of intrinsic coercivity show the most scattered data. Although no environmental conditions were reported, we know that the measurements were likely affected by temperature, humidity, measurement time constants, and field uncertainties.

\section{Conclusions}

The data presented show a path to the production of a low magnetic moment standard reference material. For fluxmetric systems, a round sample of Permalloy on a $\mathrm{Si}$ wafer seems to be a good candidate, with an estimated interlaboratory standard uncertainty of saturation flux of $3 \%$. For magnetometric systems, a possible candidate must have a highly square hysteresis loop $\left(m_{\mathrm{r}} / m_{\mathrm{s}} \approx 1\right), H_{\mathrm{c}}$ of about $400 \mathrm{~A} \cdot \mathrm{m}^{-1}(5 \mathrm{Oe})$, and $m_{\mathrm{s}} \approx 2 \times 10^{-7} \mathrm{~A} \cdot \mathrm{m}^{2}\left(2 \times 10^{4} \mathrm{emu}\right)$. Such an SRM can be made out of Permalloy films on Si substrates, which showed the best estimated interlaboratory standard uncertainty of the saturation moment of $5 \%$.

\section{Acknowledgments}

This work was done in collaboration with the National Storage Industry Consortium. We thank Ron Goldfarb, and Barry Schechtman for their consultation throughout the course of the study. We acknowledge the following individuals for their contributions to the interlaboratory comparison: J. Bain, J. Barnard, D. Bono, K. Bussman, W. Cross, T. Cumbo, W. Doyle, E. Fullerton, P. Hopkins, S. Jiang, M. Kief, M. Lederman, B. Megdal, H. Reichard, N. Rizzo, C. S. Arnold, D. Speliotis, Y. K. Liu, S. Stinnett, and D. Weller.

\section{References}

[1] J. Braun, J. P. Toennies, and C. Woll, Local layer-by-layer growth of $\mathrm{Ni}$ on hydrogen-terminated diamond $\mathrm{C}(111)$ : A combined helium-atom scattering and XPS study, Phys. Rev. B 60, 11707 (1999).

[2] M. Pitter, M. B. Hugenschmidt, and R. J. Behm, The nickel/diamond(100)-(2x1) H interface studied with electron spectroscopy, Jpn. J. Appl. Phys. I 36, 3635 (1997).

[3] Z. F. Guan, F. Deng, Q. Z. Liu, S. S. Lau, and C. A. Hewett, Nidiamond interactions, Mat. Chem. Phys. 46, 230 (1996).

[4] H. Bialas and J. Niess, Heteroepitaxial growth of nickel on diamond, Thin. Sol. Films 268, 35 (1995).

[5] D. P. Pappas, V .G. Harris, H. A. Hoff, G. L. Watena, and J. W. Glesener, Growth of single crystal copper films on diamond using fcc-iron seed layers, Mat. Res. Soc. Symp. Proc. 339, 241 (1994).

[6] H. A. Hoff, G. L. Waytena, J. W. Glesener, V .G. Harris, and D. P. Pappas, Critical thickness of single crystal fcc iron on diamond, Surf. Sci. 326, 252 (1994).

[7] D. P. Pappas, J. W. Glesener, V. G. Harris, Y. U. Idzerda, J. J. Krebs, and G. A. Prinz, Growth of fcc-Fe films on diamond, Appl. Phys. Lett 64, 28 (1994).

[8] A. W. Pacyna and K. Reubenbauer, General theory of a vibrating magnetometer with extended coild, J. Phys. E 17, 141 (1984).

[9] E. O. Samwel, T. Bolhuis, and J. C. Lodder, An alternative approach to vector vibrating sample magnetometer detection coil setup, Rev. Sci. Instrum. 69, 3204 (1998).

[10] H. J. Richter, K. A. Hempel, and J. Pfeiffer, Improvement of sensitivity of the vibrating reed magnetometer, Rev. Sci. Instrum. 59, 1388 (1988).

[11] P. J. Flanders, A vertical force alternating-gradient magnetometer, Rev. Sci. Instrum. 61, 839 (1990).

[12] M. Todorovic and S. Schultz, Miniature high-sensitivity quartz tuning fork alternating gradient magnetometry, Appl. Phys. Lett. 73, 3595 (1998)

[13] G. Tsoy, Z. Janu, M. Novak, F. Soukup, and R. Tichy, High-resolution SQUID magnetometer, Physica B, Part 2 284, 2122 (2000).

[14] G. Choe and B. Megdal, High precision magnetostriction measurement employing the B-H looper bending method, IEEE Trans. Magn., Part 2 35, 3959 (1999).

[15] International Standards Organization (ISO), Standard 57252:1994(E) 
About the authors: F. C. S. da Silva, C. M. Wang, and D. P. Pappas are physicists in the Magnetic Technology Division of the NIST Electronics and Electrical Engineering Laboratory. The National Institute of Standards and Technology is an agency of the Technology Administration, U.S. Department of Commerce. 